Revue internationale de l'économie sociale

Recma

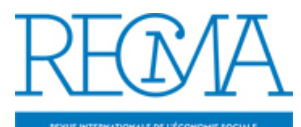

\title{
Contexte et genèse de la création des sociétés coopératives d'intérêt collectif (Scic)
}

\section{Hugues Sibille}

Numéro 324, avril 2012

URI : https://id.erudit.org/iderudit/1017781ar

DOI : https://doi.org/10.7202/1017781ar

Aller au sommaire du numéro

Éditeur(s)

Association Recma

ISSN

1626-1682 (imprimé)

2261-2599 (numérique)

Découvrir la revue

Citer cet article

Sibille, H. (2012). Contexte et genèse de la création des sociétés coopératives d'intérêt collectif (Scic). Revue internationale de l'économie sociale, (324),

110-117. https://doi.org/10.7202/1017781ar d'utilisation que vous pouvez consulter en ligne. 


\title{
CONTEXTE ET GENÈSE DE LA CRÉATION DES SOCIÉTÉS COOPÉRATIVES D'INTÉRÊT COLLECTIF (SCIC)
}

\author{
par Hugues Sibille*
}

\footnotetext{
* Délégué interministériel à l'économie sociale de juin 1998 à octobre 2001, vice-président du Crédit coopératif, président de l'Avise. Mél.: hugues.sibille@ credit-cooperatif.coop.
}

(1) Ce texte est une version remaniée du discours prononcé le 9 février 2011 par Hugues Sibille pour le dixième anniversaire des Scic, à l'invitation de la Confédération générale des Scop.

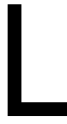
e statut de société coopérative d'intérêt collectif (Scic), établi par une loi du 17 juillet 2001, a fêté ses dix ans. Après une décennie d'existence, il intrigue et intéresse en raison de sa modernité, qui lui permet d'organiser un entrepreneuriat collectif de parties prenantes diverses ou de proposer un outil original de partenariat avec les collectivités territoriales. S'il a eu du mal à trouver sa place dans la panoplie des statuts juridiques français, il semble connaître (enfin!) le décollage que l'on attendait de lui, dans un contexte porteur pour l'économie sociale et solidaire, où les territoires inventent une nouvelle économie locale face à la mondialisation. Deux cents Scic sont agréées en mars 2012. La bonne compréhension de ce statut juridique d'entreprise coopérative implique une relecture des conditions dans lesquelles il a été élaboré et voté en juillet 2001, un siècle exactement après la loi associative de juillet 1901. La loi du 17 juillet 2001 n'est pas une loi coopérative spécifique, mais une loi ordinaire comportant un certain nombre d'articles modifiant la « loi fondamentale » des coopératives, celle de $1947^{(1)}$.

\section{Les articles spécifiques de la loi de 2001}

Avant de décrire la genèse de la loi, il est utile de rappeler les sept principaux articles qui constituent sa spécificité au regard du droit des sociétés en général et des sociétés coopératives en particulier. L'article 19 quinquies stipule que les sociétés coopératives d'intérêt collectif sont des sociétés anonymes ou des sociétés à responsabilité limitée, à capital variable, régies, sous réserve des dispositions de la présente loi, par le Code du commerce. Elles ont pour objet la production ou la fourniture de biens et de services d'intérêt collectif qui présentent un caractère d'utilité sociale. L'article 19 sexies précise que les tiers non sociétaires peuvent bénéficier des produits et des services de la Scic. L'article 19 septies détaille les différents types d'associés qu'une Scic peut réunir: les salariés de la coopérative; les personnes qui bénéficient habituellement, à titre gratuit ou onéreux, des activités de la coopérative; toute personne physique souhaitant participer bénévolement à son activité; les collectivités publiques et leurs groupements; et toute personne physique ou 
morale qui contribue par tout moyen à l'activité de la coopérative. Les Scic doivent être agréées par décision administrative dans des conditions fixées par décret (article 19 terdecies). Enfin, la décision régulièrement prise par toute société, quelle qu'en soit la forme, de modifier ses statuts pour se transformer en Scic n'entraîne pas la création d'une personne morale nouvelle (article 19 quaterdecies).

\section{Originalité de la loi}

Ces articles, insérés dans la loi de 1947, créent un nouveau statut profondément original dans le droit français.

- La Scic est clairement commerciale, puisque inscrite au registre du commerce, ce qui la distingue des associations loi 1901, qui ne le sont pas, et poursuit en même temps des finalités d'utilité sociale qui la distinguent des autres coopératives. Elle apparaît ainsi comme un " hybride » entre association et coopérative. La notion d'utilité sociale est sous contrôle de la puissance publique, via un agrément.

- C'est une coopérative loi 1947, mais ouverte à des non-sociétaires, ce qui élargit le principe de double qualité et lui permet de viser un plus large public, dans un but solidaire, altruiste. En ce sens, la Scic est bien une entreprise à but social.

- C'est le premier statut d'entreprise qui établit un multi-sociétariat dans la gouvernance même de l'entreprise, différence majeure avec un discours sur les parties prenantes (stakeholders) qui laisse in fine le pouvoir aux actionnaires (shareholders).

- Les collectivités locales peuvent être actionnaires à hauteur de $20 \%$ tout en apportant des subventions à la Scic, constituant ainsi un nouvel outil de "partenariat public-privé d'économie sociale " qui complètent l'économie mixte traditionnelle.

- Les associations qui se transforment en Scic ne créent pas une personne morale nouvelle et peuvent continuer à bénéficier de leurs avantages fiscaux ou sociaux antérieurs. L'inscription au registre du commerce ne modifie pas le caractère non lucratif de la personne morale.

\section{Contexte d'élaboration de la loi}

Les articles de la loi de 2001 n'arrivent pas par hasard, mais s'inscrivent dans le contexte politique et économique des années 1998-2000. Ils sont l'aboutissement d'un processus de travail original. Le contexte est bien résumé par le projet d'exposé des motifs, rédigé par la Délégation interministérielle à l'économie sociale (Dies) en date du 5 avril 2001. En voici des extraits: "Lors des débats parlementaires relatifs à la loi "Nouveaux services - Emplois jeunes" (octobre 1997) et à la loi relative à la lutte contre les exclusions (28 juillet 1998), une réflexion a été engagée sur la création 
d'une "entreprise à but social". La ministre de l'Emploi (Martine Aubry) en a reconnu l'intérêt et a confié à Alain Lipietz une mission d'étude sur ce sujet. Dans le même temps, le Conseil supérieur de la coopération a examiné la faisabilité d'une réponse coopérative dans ce domaine. L'un et l'autre concluent à l'intérêt de promouvoir la formule coopérative. Ils recommandent, dans le cadre de la loi de 1947, la création d'un nouveau statut, la société coopérative d'intérêt collectif. De nombreuses entreprises de ce type ont vu le jour un peu partout en Europe sous le nom de coopératives sociales. En France, il sagit d'un domaine où les associations se sont massivement investies. Le développement des activités des associations dans le champ économique, y compris dans le champ concurrentiel, est un des traits marquants de l'essor qu'elles connaissent aujourd' hui. La légitimité du rôle économique des associations est reconnue par le législateur. Le projet de Scic s'inscrit dans cette problématique. La Scic n'a pas vocation à se substituer à l'association. Elle a un caractère optionnel. " Cet extrait de l'exposé des motifs pointe cinq éléments clefs du contexte.

\section{Création et développement de nouvelles activités économiques d'utilité sociale}

Deux lois, "Nouveaux services - Emplois jeunes» et loi contre les exclusions, ont massivement encouragé, en 1997 et 1998, la création de nouvelles activités économiques d'utilité sociale correspondant à des "besoins émergents ou non satisfaits". Ces lois ont été accompagnées de programmes de développement et de budgets importants. Ainsi, 150000 emplois jeunes associatifs, à 15000 euros de financement public par emploi, soit 2,25 milliards d'euros pendant cinq ans, représentent un investissement considérable dans l'économie sociale et solidaire, terme qui n'était pas couramment utilisé à l'époque. Il s'agissait de passer d'une politique d'emploi à une politique d'activité.

Les statuts juridiques ne répondaient pas pleinement à ces nouvelles activités, d'où une étude confiée à Alain Lipietz, par lettre de mission de la ministre de l'Emploi en date du 17 septembre 1998. De son côté, la loi contre les exclusions de 1998 voulait doubler le nombre de plans locaux d'insertion par l'économique (Plie), créait des fonds départementaux d'insertion et entendait développer un « secteur mixte ».

\section{Enjeu fiscal sur les activités économiques des associations}

Les instructions fiscales de 1998, instituant la règle des $4 \mathrm{P}$ (public, produit, prix, publicité), ont mis au jour l'ampleur du sujet et commencé à clarifier le flou de la notion d'utilité sociale, en définissant des critères de fiscalisation des activités associatives à l'impôt sur les sociétés (IS), à la taxe professionnelle (TP) et à la TVA et en instituant une possible sectorisation des activités assujetties à l'impôt. La règle des $4 \mathrm{P}$ fixait un faisceau d'indices pour définir ce qu'est l'utilité sociale, après laquelle couraient depuis longtemps les associations, comme en témoignent les travaux du Conseil national de la vie associative (CNVA) de cette époque. Le faisceau 
d'indices est ainsi constitué: les activités d'utilité sociale s’adressent-elles à un public fragile? constituent-t-elles un produit différent des activités marchandes comparables? sont-elles pratiquées à des prix inférieurs à la concurrence commerciale? recourent-elles à la publicité (quatrième $\mathrm{P}$ ) commerciale? Les années 1998 et 1999 sont marquées par une intense négociation entre les pouvoirs publics et les associations, via ces règles d'imposition fiscale, sur la conception d'une socioéconomie non lucrative. Ces conceptions ont été particulièrement exprimées lors des Assises nationales de la vie associative des 20 et 21 février 1999. Ces clarifications conceptuelles et méthodologiques serviront de base pour préciser l'objet social des Scic, prévu par l'article 19 quinquies de la loi du 17 juillet 2001.

\section{Des expériences innovantes nombreuses sur le terrain}

Depuis plusieurs années apparaissent de nouvelles initiatives socioéconomiques, comme les crèches parentales, les entreprises de recyclage, les régies de quartier, les associations intermédiaires et la toute première coopérative d'activité (Cap services), ainsi que de nouveaux outils de financement comme France active et l'engagement de la Caisse des dépôts dans ce secteur émergent. Ces expériences se heurtent à un cadre juridique mal adapté.

\section{Des dynamiques régionales nouvelles}

En 1999 et 2000 ont lieu les consultations régionales de l'économie sociale et solidaire. C'est la première fois que ces deux adjectifs sont accolés. Les préfets sont mobilisés pour organiser ces consultations et découvrent alors le poids socioéconomique de cette économie sociale et solidaire qu' ils ignoraient. Les consultations, auxquelles 5000 personnes participent sur tout le territoire, vont générer un décloisonnement des acteurs sur le terrain et des dynamiques territoriales propices à de nouvelles activités économiques d'utilité sociale, qui se poursuivent aujourd'hui à travers les chambres régionales. Alain Lipietz participe à ces consultations et s'en imprègne pour rédiger son rapport.

\section{Des lois sur les coopératives et entreprises sociales partout en Europe}

La loi sur les Scic s'inscrit, enfin, dans un contexte international porteur, avec de nombreux pays qui nous ont précédés dans des législations spécifiques. Les plus connues sont les coopératives sociales italiennes, impliquées dans la fourniture de services sociaux et de santé, mais aussi dans l'insertion par le travail des personnes défavorisées. Elles ont été consacrées par une loi de 1991. En 1999, au moment du débat sur les Scic en France, il existe 4300 coopératives sociales en Italie, ce qui est considérable.

En Espagne, une loi du 16 juillet 1999 a créé des coopératives d'initiative sociale; de même au Portugal, par une loi du 15 janvier 1998. La Belgique s'est singularisée en créant la société à finalité sociale (SFS), qui sera regardée de près par les rédacteurs de la loi sur les Scic. La loi belge a visé les activités d'insertion et les services de proximité. Elle n’a pas créé de statut 
sui generis d'entreprise, mais une modalité particulière qui peut être insérée dans les statuts de toute société commerciale, à condition qu'elle respecte des conditions relatives au but social, aux bénéfices et à la limitation des droits de vote par rapport au capital. Cette toile de fond internationale jouera un rôle important dans l'élaboration de la législation sur les Scic. Nombre des acteurs étaient imprégnés de l'exemple italien.

\section{Un processus original d'élaboration}

L'émergence des Scic et la préparation de la loi se font en lien étroit avec le terrain, avec la réalité entrepreneuriale concrète, et pas seulement en chambre, que ce soit celle des députés, de l'administration ou des institutions de l'économie sociale.

\section{La démarche collective d'innovation (DCI)}

La Confédération générale des Scop (CGScop) a d'abord poursuivi une étude-action sur les modifications à apporter à la loi de 1947, suivie d'une expérimentation baptisée " démarche collective d'innovation " (DCI), de juillet 2000 à décembre 2001. Cette DCI visait à sélectionner des projets de Scic, à les accompagner dans le cadre d'une expérimentation nationale et à participer à l'élaboration du texte de loi. La DCI comportait trois volets: l'accompagnement des projets par la formation et la mise en réseau des porteurs; un groupe de réflexion et des séminaires pour approfondir des thèmes comme la mixité des ressources, le tiers secteur et les externalités positives; la création d'un inter-réseau Scic, permettant l'échange de bonnes pratiques.

Dix-sept projets de terrain ont effectivement participé à la DCI, cinq cents ayant manifesté un intérêt, oeuvrant dans les secteurs suivants: l'environnement, la culture, les crèches parentales, le développement local, la gestion de l'espace rural, l'éducation, les services de proximité, l'organisation de filières (bois, pierre, nouvelles technologies de l'information et de la communication [NTIC]).

Ce qui frappe dans ces projets, c'est leur ancrage territorial et leurs liens avec le programme " Nouveaux services - Emplois jeunes». La DCI a permis de faire travailler ensemble des projets entrepreneuriaux de terrain, des têtes de réseau, des financeurs et les pouvoirs publics. Ces réunions mettaient en présence, aux côtés de la CGScop, la Délégation interministérielle à l'économie sociale, la Direction générale de l'emploi et de la formation professionnelle (DGEFP), le ministère de l'Agriculture, la Datar, la Caisse des dépôts, France active, l'Institut de développement de l'économie sociale (Ides), la Macif... Parmi les réseaux, furent notamment actifs les coopératives d'utilisation du matériel agricole (Cuma), les crèches parentales, les foyers de jeunes travailleurs, les entreprises d'insertion, etc. Ce travail collectif a permis une coproduction de l'entreprise Scic et de la loi, lui donnant son statut spécifique. 


\section{Un groupe mixte de préparation de la loi}

La préparation de la loi pouvait ainsi s'appuyer sur une série importante de travaux: le rapport Lipietz, les travaux du Conseil supérieur de la coopération (CSC), la DCI, le rapport des consultations régionales, les travaux préparatoires à la loi "Nouveaux services - Emplois jeunes", la règle des $4 \mathrm{P}$. La matière n'était pas simple pour autant. En particulier, l'articulation entre le statut de Scic et les associations loi 1901 était difficile. Le monde associatif redoutait que l'on n'oblige les associations à changer de statut quand elles auraient des activités économiques. Elles craignaient une marchandisation progressive des associations. Les fédérations associatives appréhendaient une fuite de leurs adhérents vers des fédérations coopératives. Pour cette raison, le CNVA remit en mars 2001 un avis témoignant de certaines craintes et rappelant son attachement au statut associatif.

La Délégation interministérielle mit en place (sans doute pour la première fois) un groupe composé de représentants du monde coopératif (Groupement national de la coopération [GNC], aujourd'hui Coop FR, et CGScop), du monde associatif (CPCA, CNVA) et des administrations (Dies, DGEFP, direction régionale du travail, direction des entreprises, législation fiscale, affaires civiles...). Ce groupe mixte permit de lever, d'un côté, les craintes des associations, qui redoutaient, outre le caractère obligatoire de transformation en Scic, les pertes d'agréments et d'avantages publics antérieurs, et, de l'autre, les réserves du GNC sur une possible remise en cause par la loi Scic des principes coopératifs "de base ", la double qualité et la ristourne.

\section{Portage politique de la loi: création d'un secrétariat d'Etat}

Si le projet de loi put aller à son terme, ce fut aussi parce qu'il bénéficia d'un portage politique favorable. En mars 2000, le Premier ministre, Lionel Jospin, lors d'un remaniement ministériel, avait créé un secrétariat d'Etat àl'Economie solidaire et l'avait confiéà un écologiste, Guy Hascoët. Il s'agissait d'une opération politique en direction des écologistes, prolongeant la mission confiée à un autre écologiste, Alain Lipietz, et prenant acte de la montée politique de ce courant d'opinion sur le sujet de l'économie solidaire en région.

La création du secrétariat d'Etat donnait une visibilité à l'ESS et de nouveaux moyens politiques pour pousser les projets. C'est ce qui se produisit avec la loi sur les Scic. On peut se demander si, sans la présence d'un secrétariat d'Etat, le projet de loi serait allé à son terme, car c'était la fin de la mandature et l'agenda parlementaire était saturé. Le projet Scic n'avait ainsi pu être inséré dans la loi de modernisation sociale, mais Matignon accepta d'insérer les articles dans la loi 2001-624 du 17 juillet 2001 portant " diverses mesures d'ordre social, éducatif et culturel ».

Le texte passa en quelque sorte aux forceps. Lors du débat au Sénat, le rapporteur, Jean-Louis Lorrain, reprocha la "précipitation" du gouvernement sur un texte "qui n'était passé ni en Conseil d'Etat, ni en Conseil 
desministres» et demanda qu'il fasse l'objet d'une loi spécifique. Le texte fut finalement adopté, mais un recours fut déposé devant le Conseil constitutionnel, qui le classa sans suite.

\section{Conclusion}

(2) La loi 2012-387 du 22 mars relative à la simplification et à l'allégement des démarches administratives a supprimé cet agrément préfectoral. Voir " En bref " dans ce numéro. NDLR.
L'élaboration du cadre relatif aux Scic, en tant que concept et en tant que statut juridique, est empreinte d'un fort niveau d'innovation sociale. Sur le fond, la Scic crée, de facto et de jure, une nouvelle forme d'entreprise, commerciale, mais d'intérêt collectif, reposant sur le multi-sociétariat des parties prenantes. Pour la première fois, des logiques d'intérêt qui peuvent être parfois contradictoires, celles des usagers, celles des salariés, celles de la communauté locale..., se retrouvent dans la gouvernance même de l'entreprise. En ce sens, la Scic invente une entreprise territoriale nouvelle, qui permet à des acteurs différents de faire converger leurs logiques pour développer le territoire: produire des énergies renouvelables, permettre la mobilité, organiser des circuits courts, structurer une filière. Dans le même temps, la possible présence des collectivités locales jusqu'à $20 \%$ du capital (qui fut arraché au ministère de l'Intérieur en 2001) fait de la Scic une plateforme potentielle de partenariats public-privé dans des secteurs comme la santé, la culture, l'éducation, etc. La Scic peut présenter une dimension moins administrée, plus entrepreneuriale, que la société d'économie mixte (SEM).

Sur le plan de la méthode, cette loi résulte d'un processus innovant, articulant des expérimentations de terrain, des formulations conceptuelles et de la créativité juridique. C'est une « coproduction législative ». Ce caractère innovant et original de l'élaboration des Scic peut être tempéré par trois questionnements.

Le premier, c'est que, pour des raisons d'équilibre entre monde associatif et monde coopératif, mais aussi pour des raisons liées au "génie administratif » français, le texte final s'est révélé assez complexe, avec une conception administrative forte. Alain Lipietz avait suggéré que les agréments soient remis par les pairs, c'est-à-dire par le secteur lui-même. Ce fut finalement un agrément préfectoral. Ce caractère assez administratif a peut-être freiné un développement plus rapide des Scic. La complexité aura été jusqu'ici le principal obstacle à leur développement. La question de la simplification reste posée ${ }^{(2)}$.

Le deuxième est que, face à cette complexité et à des craintes exprimées, en particulier par les associations à l'époque, il n'y a pas eu d'incitation, fiscale ou autre, à choisir ce statut. De nombreux projets ont flirté avec l'idée de choisir le statut Scic, mais sont finalement restés dans des statuts mieux connus, car il n'y avait pas d'avantage économique à faire ce choix. Le troisième tient au fait que, une fois la loi votée, les moyens pour populariser le statut des Scic, accompagner les porteurs de projet ou créer des Scic sont restés limités. Un nouveau gouvernement se mit en place, 
quin'avait pas voté cette loi et ne la mit pas en avant. Les mouvements coopératifs et associatifs eux-mêmes furent prudents pour créer de nouvelles coopératives ou transformer des associations en Scic. Du coup, la montée en puissance des Scic a été plus lente que celle espérée.

Une décennie plus tard, cela a changé. L'entrepreneuriat social et d'économie sociale connaît un regain d'intérêt. Nul doute que ce statut d'entreprise, simplifié et accompagné, ne connaisse un vrai succès dans les années à venir. Il reste d'une profonde modernité. 